\title{
Influence of the COVID-19 Pandemic and Bystander- victim Relationship on the Willingness of Thai Healthcare Providers and Laypersons to Perform Cardiopulmonary Resuscitation: a Cross-sectional Online Survey
}

\author{
Suhattaya Boonmak \\ Khon Kaen University \\ Thapanawong Mitsungnern \\ Khon Kaen University \\ Pimmada Boonmak \\ Khon Kaen Wittayayon school \\ Polpun Boonmak ( $\boldsymbol{\sigma}$ bpolpu@kku.ac.th ) \\ Khon Kaen University
}

\section{Research Article}

Keywords: Basic cardiac life support, cardiopulmonary resuscitation, COVID-19 pandemic, life support care, willingness

Posted Date: August 26th, 2021

DOI: https://doi.org/10.21203/rs.3.rs-702605/v1

License: (c) (i) This work is licensed under a Creative Commons Attribution 4.0 International License. Read Full License 


\section{Abstract}

Background: Out-of-hospital cardiac arrest (OHCA) is a significant health problem in many Asian countries. Bystander cardiopulmonary resuscitation (CPR) can reduce mortality from OHCA. The willingness of bystanders to initiate aid is also critical. During the COVID-19 pandemic, CPR eases to spread of COVID-19 with negatively affect the decision. The objectives were to study the influence of the COVID-19 pandemic and bystander-victim relationship on the willingness of Thai healthcare providers (HCPs) and laypersons to perform CPR and associated factors.

Methods: The cross-sectional online survey was conducted between August to November 2020 in a Thai population. A structured questionnaire was developed and given to volunteers as an online survey. We recorded participant characteristics and willingness to perform compression-only CPR (W-COC) and conventional CPR (W-CC) on family members, acquaintances, and strangers during the pandemic and in a non-pandemic situation and analyzed associated factors.

Results: We included 419 laypersons and 716 HCPs. Lay-participants expressed less W-COC on acquaintances and strangers (risk ratio [RR] 0.74-0.85), but not on family members. By contrast, the HCPs were less W-COC across the board (RR 0.84-0.92). Both groups were less W-CC on all recipients (RR 0.430.54). There was no difference in participants' W-COC and W-CC on family members and acquaintances, except that HCPs expressed greater W-CC on family members. Participants in all groups were less W-COC and W-CC on strangers. W-COC was correlated with CPR knowledge (Odds ratio [OR] 2.32), self-efficacy (OR 1.96), single marital status (OR 1.91), and being an HCP (OR 2.00). W-CC on family members was related to CPR knowledge (OR 2.16).

Conclusion: Participants were less willing during the COVID-19 pandemic on all groups except family members of lay participants. The victim's relationship to the participant was more important in conventional CPR than compression-only CPR, especially in HCPs. CPR knowledge, self-efficacy, single marital status, and being an HCP related to higher willingness.

Trial registration: TCTR20210330003 (Thai Clinical Trials Registry)

\section{Background}

Out-of-hospital cardiac arrest (OHCA) is a significant health problem in many Asian countries. ${ }^{1,2}$ Bystander CPR is a type of first aid that aims to manage cardiac and respiratory arrest until the victim can be sent to a medical facility. ${ }^{3,4}$ Bystander CPR plays a vital role in improving survival to hospital discharge in OHCA victims. ${ }^{2,5}$ There are two currently accepted bystander CPR techniques: conventional CPR and chest compression-only CPR. ${ }^{3,4}$ Conventional $\mathrm{CPR}$ requires the knowledge and skills to perform an initial assessment, chest compression, and ventilation and to use an automated defibrillator (AED). Chest compression-only CPR (compression-only CPR; hands-only CPR) is an alternative technique that is easier to learn and remember, thus increasing the likelihood that CPR will be performed. This technique consists of initial assessment, chest compression, and AED without ventilation. Conventional CPR is recommended for 
health care providers (HCPs) and laypeople trained and willing to give rescue breaths and chest compression. ${ }^{3}$

Although knowledge and skills are key factors in whether CPR is performed, the willingness of bystanders to initiate aid is also critical. Studies in various parts of Asia have found that anywhere from $10.5-79.0 \%$ of $\mathrm{OHCA}$ victims in the region receive bystander $\mathrm{CPR},{ }^{1,2,6}$ and willingness to perform bystander CPR ranges 10.3-98.6\%. ${ }^{7-12}$ Willingness to perform CPR is affected by various complex factors having to do with the bystander (experience, training, age, fear, self-efficacy, physical fitness), technique (ventilation or nonventilation), the victim (age, gender, relationship), and the environment (location, country, safety, infection risk). ${ }^{8-9,11,13-15}$ It seems likely that the possibility of airborne transmission of Coronavirus disease 2019 (COVID-19) during ventilation and chest compression would negatively affect the willingness of bystanders to perform CPR. However, previous studies and a systematic review have yielded inconclusive data, especially in Asian countries. ${ }^{16-18}$ In addition, considering the central role of the family and interpersonal relationships in many Asian cultures, ${ }^{19-20}$ the degree of intimacy between bystanders and victims (such as whether they are family members, acquaintances, or strangers) may affect the decision to perform CPR. Previous studies have shown that bystanders are generally more willing to perform CPR on a family member than a stranger. ${ }^{7-11}$ In our research, we conducted an anonymous online survey to study the willingness of both laypeople and HCPs to perform bystander CPR in Thailand, a low-risk country for COVID-19, as well as associated factors.

\section{Methods}

\section{Study design}

This prospective descriptive study was based on a cross-sectional online survey conducted between Aug 31 and Nov 30, 2020, in Thailand. We obtained Institute Review Board approval (HE631328) before commencing the study. The authors designed a structured questionnaire based on those described in the

previous studies $^{9-12}$, with the content adjusted for use in a Thai context. We conducted a pilot study with 30 participants to check the reliability of the questionnaire, which was then modified based on the results. We then had three experts in the content area test the content validity (content validity index 0.95). The final questionnaire showed acceptable reliability, with a Cronbach's alpha coefficient of $0.86(95 \% \mathrm{Cl} 0.78-0.92)$. This study is also reported in accordance with the STrengthening the Reporting of OBservational studies in Epidemiology (STROBE) guideline.

\section{Questionnaire}

Participants filled out an electronic consent form before taking the questionnaire. We did not record names, e-mail addresses, or phone numbers in order to maintain anonymity. The questionnaire consisted of two sections: participant characteristics and willingness to perform bystander CPR. The former consisted of 10 questions to determine gender, age, marital status, education level, occupation of health care providers (such as a doctor, dentist, pharmacist, nurse, medical technician, physical therapist, or physician assistant), knowledge and experience in performing CPR, previous CPR training, and CPR self-efficacy. Participants were allowed to skip any questions to which they did want to respond. We evaluated CPR knowledge using 
five questions. If a participant answered all five correctly, they were considered to have "good" CPR knowledge. Willingness to perform bystander CPR was assessed for seven techniques: compression-only CPR, conventional CPR (whether it be mouth-to-mouth, face shield, or pocket mask), chest compression, $A E D$, mouth-to-mouth ventilation, mouth-to-face shield ventilation, and mouth-to-pocket mask ventilation. Participants were asked to indicate their willingness to perform each technique on a family member, acquaintance, and stranger. A family member was defined as either a close relative of the participant (spouse, parent, grandparent, sibling, child) or a member of their extended family (aunt, uncle, cousin, nephew, niece, or sibling-in-law). An acquaintance was defined as someone the participant knows personally/socially. The situation was phrased as follows: "Imagine you came across an unconscious person in a restaurant during the current pandemic. Would you perform CPR? How about in a time when there is no pandemic?" Possible answers were "yes," "no," and "not sure." A "yes" answer was classified as willing to perform CPR and "no" or "not sure" as unwilling. The questionnaire included CPR information such as pictures and descriptions in order to assist participants in deciding. The questionnaire took approximately 15 minutes to complete.

\section{Data collection}

Volunteers were recruited using an online survey (Google Forms, Google Inc.) distributed through the public health office of every province in Thailand, official e-mail contact lists of universities, including alumni associations and schools. The authors also shared the survey through their e-mail contact lists. The first page of the survey described the purpose of the study and information about participation (including the withdrawal right). A link to the questionnaire was included in the survey and could only be accessed after the participant agreed to participate. We sent out a reminder to our contacts to encourage them to fill out our survey.

\section{Statistical analysis}

Data analysis was performed using SPSS Statistics for Windows version 26.0 (IBM Corp, Armonk, NY). Subgroup analysis was performed for lay participants and HCPs. We used descriptive statistics to describe participant characteristics. Categorical data were summarized as percentage and frequency and continuous data as mean and standard deviation. We calculated proportions based on the number of participants with non-missing data. We compared pandemic and non-pandemic responses using the chi-square test and calculated a risk ratio with a $95 \%$ confidence interval. We compared willingness to perform CPR on family members, acquaintances, and strangers using two proportion $Z$ tests and calculated a difference in proportion with a $95 \%$ confidence interval or $\mathrm{p}$-value, as appropriate. All associated factors were considered as dichotomous variables except some variables such as age, education level, and work status, which were polytomous variables. Univariable analysis was used to assess the associations between pairs of categorical variables (chi-square test or Fisher's exact test appropriately), then multivariate analysis via logistic regression by an enter method with all associated factors that $p$-value were less than 0.2. Results were reported as odd's ratios with $95 \%$ confidence intervals. The estimated required sample size was calculated based on a $95 \%$ confidence level, type I error of 0.05 , the estimated prevalence of willingness to 
perform compression-only CPR based on previous studies, absolute precision of 0.10 , and power of $80 \%{ }^{7}$, $9-10,16$

\section{Results}

\section{Participant characteristics}

Because we were not able to accurately determine the total number of people who received a link to the questionnaire, it was not possible to calculate the response rate. We included 1,195 participants -419 (35.1\%) laypeople and 716 (59.9\%) HCPs. Sixty (5\%) respondents were excluded because we could not determine their classification. There were no missing data. The layperson group was $65.3 \%$, female, while the HCP was $75.1 \%$, female. The mean (SD) age was 37.3 (14.5) years in the layperson group and 39.9 (11.4) years in the HCP group. Most of the participants had completed a bachelor's degree and were working full time. In the layperson group, $65.4 \%$ of participants had heard of CPR, while almost all in the HCP group had. About half of the lay participants had taken a CPR course, compared to nearly all of the HCPs. Half of the HCPs answered questions about CPR knowledge correctly, compared to $14.1 \%$ of lay participants. Most HCPs had experience performing CPR, whereas most lay participants did not. The percentage of participants who expressed CPR self-efficacy was $13.6 \%$ and $72.8 \%$ in the lay and HCP group, respectively (Table 1). 
Table 1

Participant characteristics

\begin{tabular}{|c|c|c|c|}
\hline \multicolumn{2}{|l|}{ Variables } & $\begin{array}{l}\text { Laypersons } \\
(\mathrm{N}=419)\end{array}$ & $\begin{array}{l}\text { Health care providers } \\
(\mathrm{N}=716)\end{array}$ \\
\hline \multirow[t]{3}{*}{ Gender $(\mathrm{n},(\%))$} & male & $135(32.2)$ & $163(22.8)$ \\
\hline & female & $274(65.3)$ & $538(75.1)$ \\
\hline & not specified & $10(2.5)$ & $15(2.1)$ \\
\hline \multicolumn{2}{|l|}{ Age (year; mean (SD)) } & $37.3(14.5)$ & $39.9(11.4)$ \\
\hline \multirow[t]{3}{*}{ Marital status (n, (\%)) } & single & $191(45.6)$ & $4.5(56.6)$ \\
\hline & married & $207(49.4)$ & $272(38.0)$ \\
\hline & not specified & $21(5.0)$ & $39(5.4)$ \\
\hline \multirow[t]{5}{*}{ Education level (n, (\%)) } & primary education or under & $4(1.0)$ & $0(0.0)$ \\
\hline & secondary education & $83(19.7)$ & $15(2.1)$ \\
\hline & bachelor's degree & $180(43.0)$ & $467(65.2)$ \\
\hline & master's degree or higher & $135(32.2)$ & $213(29.8)$ \\
\hline & not specified & $17(4.1)$ & $21(2.9)$ \\
\hline \multirow[t]{6}{*}{ Work status (n, (\%)) } & full time ( $\geq 30$ hours) & $241(57.5)$ & $616(86.0)$ \\
\hline & part-time (<30 hours) & $24(5.7)$ & $23(3.2)$ \\
\hline & retired & $20(4.8)$ & $19(2.7)$ \\
\hline & unemployed & $21(5.0)$ & $4(0.6)$ \\
\hline & student & $60(14.3)$ & $28(3.8)$ \\
\hline & not specified & $53(12.7)$ & $26(3.6)$ \\
\hline \multicolumn{2}{|l|}{ Awareness of CPR $(n,(\%))$} & $274(65.4)$ & 708 (98.9) \\
\hline \multicolumn{2}{|l|}{ CPR training $(\mathrm{n},(\%))$} & $191(45.6)$ & 707 (98.7) \\
\hline \multicolumn{2}{|l|}{ CPR knowledge (n, (\%)) } & $59(14.1)$ & $401(56.0)$ \\
\hline \multicolumn{2}{|c|}{ Experience performing CPR $(n,(\%))$} & $51(12.2)$ & $541(75.6)$ \\
\hline \multicolumn{2}{|l|}{ CPR Self-efficacy $(\mathrm{n},(\%))$} & $57(13.6)$ & $521(72.8)$ \\
\hline
\end{tabular}


Lay participants were less willing to perform compression-only CPR on acquaintances and strangers during the COVID-19 pandemic than during a non-pandemic period, but this was not the case for family members. Healthcare professionals were less willing to perform compression-only CPR during the pandemic regardless of their relationship with the victim. Participants in both groups were less willing to perform conventional CPR regardless of relationship (Table 2). 
Table 2

Impact of the COVID-19 pandemic and the relationship between the bystander and victim on willingness to perform compression-only and conventional CPR

\begin{tabular}{|c|c|c|c|c|c|c|}
\hline \multirow[t]{3}{*}{ Variable } & \multirow{3}{*}{$\begin{array}{l}\text { Family } \\
\text { members }\end{array}$} & \multirow[t]{3}{*}{ Acquaintances } & \multirow[t]{3}{*}{ Strangers } & \multicolumn{3}{|c|}{ The difference in proportions $(95 \% \mathrm{Cl})$} \\
\hline & & & & $\begin{array}{l}\text { Between } \\
\text { family } \\
\text { member }\end{array}$ & $\begin{array}{l}\text { Between } \\
\text { family } \\
\text { member }\end{array}$ & $\begin{array}{l}\text { Between } \\
\text { acquaintance }\end{array}$ \\
\hline & & & & $\begin{array}{l}\text { and } \\
\text { acquaintance }\end{array}$ & $\begin{array}{l}\text { and } \\
\text { stranger }\end{array}$ & \\
\hline \multicolumn{7}{|c|}{ Willingness to perform compression-only CPR } \\
\hline \multicolumn{7}{|c|}{ Laypersons $(\mathrm{N}=419)$} \\
\hline $\begin{array}{l}\text { Non- } \\
\text { pandemic } \\
(\mathrm{n},(\%))\end{array}$ & $205(48.9)$ & $213(50.8)$ & $\begin{array}{l}186 \\
(44.4)\end{array}$ & $1.9(-4.9,8.7)$ & $\begin{array}{l}4.5(-1.2 \\
10.2)\end{array}$ & $\begin{array}{l}6.4(-0.4 \\
13.2)\end{array}$ \\
\hline $\begin{array}{l}\text { Pandemic } \\
(\mathrm{n},(\%))\end{array}$ & $193(46.1)$ & $182(43.4)$ & $\begin{array}{l}138 \\
(32.9)\end{array}$ & $2.7(-4.0,9.4)$ & $\begin{array}{l}13.2 \\
(6.6, \\
19.8)\end{array}$ & $\begin{array}{l}10.5(3.9 \\
17.1)\end{array}$ \\
\hline $\begin{array}{l}\text { Risk ratio } \\
(95 \% \mathrm{Cl})\end{array}$ & $\begin{array}{l}0.94 \\
(0.82- \\
1.09)\end{array}$ & $\begin{array}{l}0.85(0.74- \\
0.99)\end{array}$ & $\begin{array}{l}0.74 \\
(0.62- \\
0.88)\end{array}$ & & & \\
\hline \multicolumn{7}{|c|}{ Health care providers $(n=716)$} \\
\hline $\begin{array}{l}\text { Non } \\
\text { pandemic } \\
(\mathrm{n},(\%))\end{array}$ & $645(90.1)$ & $640(89.4)$ & $\begin{array}{l}615 \\
(85.9)\end{array}$ & $\begin{array}{l}0.7(-2.4, \\
0.8)\end{array}$ & $\begin{array}{l}4.2(0.8 \\
7.6)\end{array}$ & $3.5(0.9,6.9)$ \\
\hline $\begin{array}{l}\text { Pandemic } \\
(\mathrm{n},(\%))\end{array}$ & $591(82.5)$ & $561(78.4)$ & $\begin{array}{l}517 \\
(72.2)\end{array}$ & $4.1(-0.1,8.2)$ & $\begin{array}{l}10.3 \\
(6.0 \\
14.6)\end{array}$ & $\begin{array}{l}6.2(1.7 \\
10.7)\end{array}$ \\
\hline $\begin{array}{l}\text { Risk ratio } \\
(95 \% \mathrm{Cl})\end{array}$ & $\begin{array}{l}0.92 \\
(0.88- \\
0.96)\end{array}$ & $\begin{array}{l}0.88(0.84- \\
0.92)\end{array}$ & $\begin{array}{l}0.84 \\
(0.80- \\
0.89)\end{array}$ & & & \\
\hline
\end{tabular}

\section{Willingness to perform conventional CPR}

Laypersons $(\mathrm{N}=419)$

$\begin{array}{lllllll}\text { Non } & 180(43.0) & 166(39.6) & 118 & 3.4(-3.3, & 14.8 & 11.4(5.0, \\ \text { pandemic } & & & (28.2) & 10.1) & (8.3, & 17.8) \\ (\mathrm{n},(\%)) & & & & & 21.3) & \end{array}$

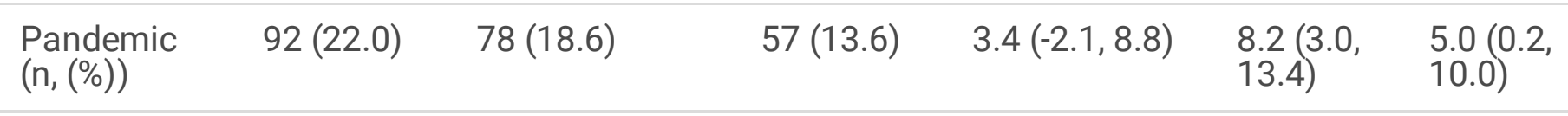

$\begin{array}{llll}\text { Risk ratio } & 0.51 & 0.47(0.37- & 0.48 \\ (95 \% \mathrm{Cl}) & (0.41- & 0.59) & (0.36- \\ & 0.63) & & 0.64)\end{array}$

Health care providers $(n=716)$ 


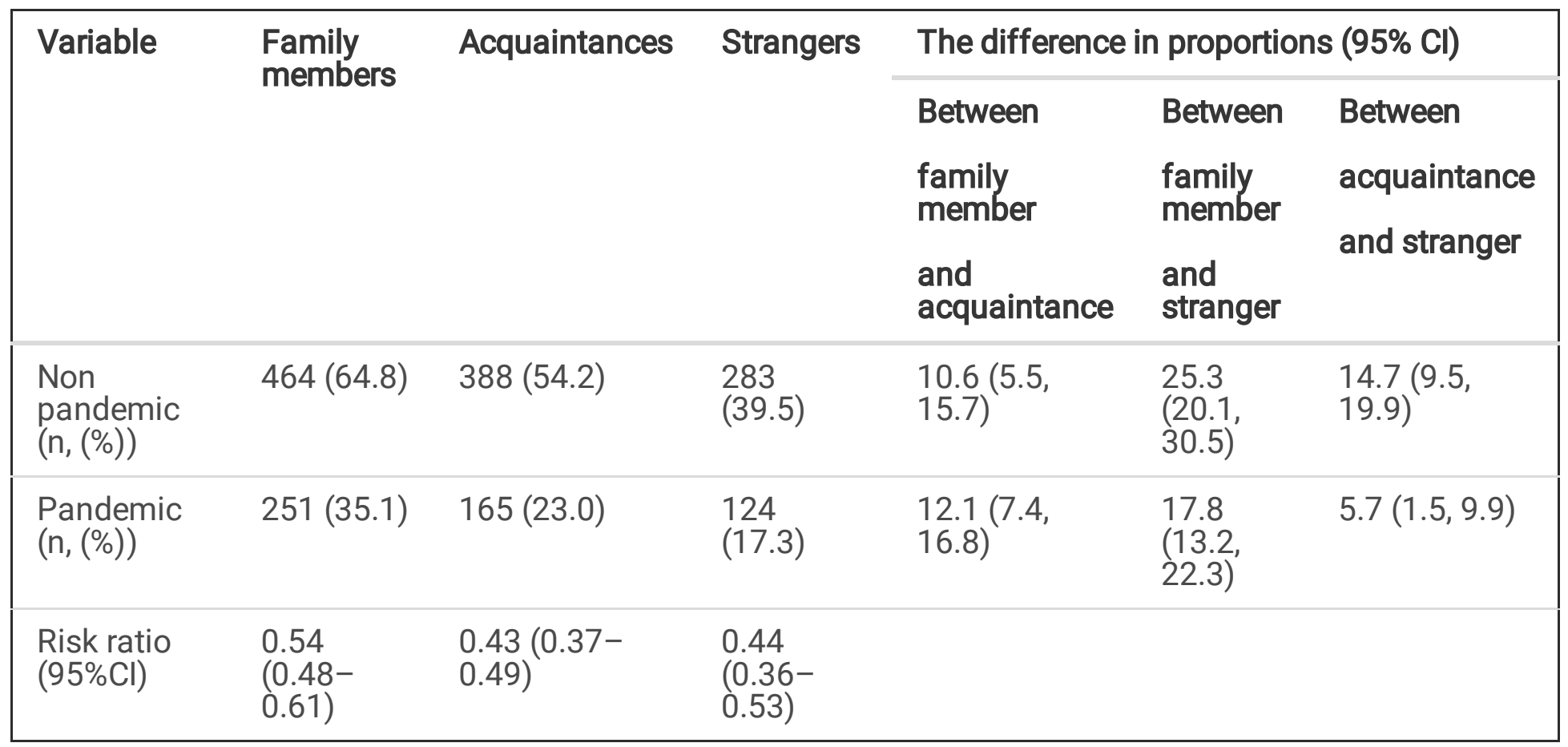

Table 2 also compares participants' willingness to perform CPR during the pandemic by relationship to the victim. There was no difference in either group in participants' willingness to perform compression-only CPR between family members and acquaintances. However, HCPs were more willing to perform conventional CPR for a family member than for an acquaintance. Participants in both groups were more willing to CPR of any type on acquaintances than on strangers.

As shown in Table 3, participants with more CPR knowledge, self-efficacy, and experience were more likely to be willing to perform compression-only CPR regardless of relationship, as were HCPs. Single marital status was related to a higher willingness to perform compression-only CPR on family members and acquaintances. Good CPR knowledge was associated with a higher willingness to perform conventional CPR on family members. 
Table 3

Factors associated with willingness to perform compression-only and conventional CPR (only those with statistical significance)

\begin{tabular}{|c|c|c|c|c|c|c|}
\hline \multirow{3}{*}{$\begin{array}{l}\text { Associated } \\
\text { factors }\end{array}$} & \multicolumn{6}{|c|}{ Odds ratio (95\% Cl) } \\
\hline & \multicolumn{2}{|c|}{ Family members } & \multicolumn{2}{|c|}{ Acquaintances } & \multicolumn{2}{|l|}{ Strangers } \\
\hline & Univariable & Multivariable & Univariable & Multivariable & Univariable & Multivariable \\
\hline \multicolumn{7}{|c|}{ Willingness to perform compression-only CPR } \\
\hline \multicolumn{7}{|c|}{ Age (control factor: $20-40$ years old) } \\
\hline $\begin{array}{l}-<20 \\
\text { years old }\end{array}$ & NS & NS & $\begin{array}{l}0.25 \\
(0.11- \\
0.58)\end{array}$ & NS & NS & NS \\
\hline $\begin{array}{l}-41-60 \\
\text { years old }\end{array}$ & $\begin{array}{l}2.72 \\
(2.39- \\
3.12)\end{array}$ & NS & $\begin{array}{l}1.57 \\
(1.38- \\
1.80)\end{array}$ & NS & $\begin{array}{l}2.52 \\
(2.20- \\
2.90)\end{array}$ & NS \\
\hline $\begin{array}{l}->60 \\
\text { years old }\end{array}$ & $\begin{array}{l}1.78 \\
(1.54- \\
2.04)\end{array}$ & NS & $\begin{array}{l}1.20 \\
(1.06- \\
1.37)\end{array}$ & NS & $\begin{array}{l}1.88 \\
(1.64- \\
2.15)\end{array}$ & NS \\
\hline \multicolumn{7}{|c|}{ Education (control factor: middle school) } \\
\hline $\begin{array}{l}\text { - High } \\
\text { school }\end{array}$ & $\begin{array}{l}3.50 \\
(2.26- \\
5.42)\end{array}$ & NS & $\begin{array}{l}2.21 \\
(1.43- \\
3.40)\end{array}$ & NS & $\begin{array}{l}2.49 \\
(1.61- \\
3.85)\end{array}$ & NS \\
\hline $\begin{array}{l}\text { - } \\
\text { Bachelor's } \\
\text { degree }\end{array}$ & $\begin{array}{l}2.65 \\
(1.67- \\
4.19)\end{array}$ & NS & $\begin{array}{l}1.94 \\
(1.23- \\
3.06)\end{array}$ & NS & $\begin{array}{l}1.98 \\
(1.25- \\
3.13)\end{array}$ & NS \\
\hline \multicolumn{7}{|c|}{ Work status (control factor: full time) } \\
\hline - Part-time & $\begin{array}{l}2.58 \\
(1.92- \\
3.46)\end{array}$ & NS & NS & NS & $\begin{array}{l}2.78 \\
(2.03- \\
3.81)\end{array}$ & NS \\
\hline - Student & $\begin{array}{l}0.64 \\
(0.64- \\
0.65)\end{array}$ & $\begin{array}{l}0.24(0.16- \\
0.37)\end{array}$ & $\begin{array}{l}1.64 \\
(1.57- \\
1.68)\end{array}$ & NS & NS & NS \\
\hline $\begin{array}{l}\text { Single } \\
\text { marital } \\
\text { status }\end{array}$ & $\begin{array}{l}1.32 \\
(1.02- \\
1.71)\end{array}$ & $\begin{array}{l}1.91(1.30- \\
2.81)\end{array}$ & $\begin{array}{l}1.40 \\
(1.09- \\
1.80)\end{array}$ & $\begin{array}{l}1.88(1.32- \\
2.70)\end{array}$ & NS & NS \\
\hline
\end{tabular}

NS = not statistically significant 


\begin{tabular}{|c|c|c|c|c|c|c|}
\hline \multirow{3}{*}{$\begin{array}{l}\text { Associated } \\
\text { factors }\end{array}$} & \multicolumn{6}{|c|}{ Odds ratio $(95 \% \mathrm{Cl})$} \\
\hline & \multicolumn{2}{|c|}{ Family members } & \multicolumn{2}{|c|}{ Acquaintances } & \multicolumn{2}{|l|}{ Strangers } \\
\hline & Univariable & Multivariable & Univariable & Multivariable & Univariable & Multivariable \\
\hline $\begin{array}{l}\text { Awareness } \\
\text { of CPR }\end{array}$ & $\begin{array}{l}4.74 \\
(3.34- \\
6.74)\end{array}$ & NS & $\begin{array}{l}4.37 \\
(3.07- \\
6.23)\end{array}$ & NS & $\begin{array}{l}4.54 \\
(3.12- \\
6.59)\end{array}$ & NS \\
\hline $\begin{array}{l}\mathrm{CPR} \\
\text { training }\end{array}$ & $\begin{array}{l}4.49 \\
(3.34- \\
6.05)\end{array}$ & NS & $\begin{array}{l}4.23 \\
(3.14- \\
5.69)\end{array}$ & NS & $\begin{array}{l}5.17 \\
(3.78- \\
7.08)\end{array}$ & NS \\
\hline $\begin{array}{l}\text { CPR } \\
\text { experience }\end{array}$ & $\begin{array}{l}3.26 \\
(2.50- \\
4.25)\end{array}$ & $\begin{array}{l}0.60(0.36- \\
0.99)\end{array}$ & $\begin{array}{l}3.04 \\
(2.36- \\
3.93)\end{array}$ & NS & $\begin{array}{l}3.50 \\
(2.74- \\
4.49)\end{array}$ & NS \\
\hline $\begin{array}{l}\text { Health } \\
\text { care } \\
\text { provider }\end{array}$ & $\begin{array}{l}5.34(4.07- \\
7.00)\end{array}$ & $\begin{array}{l}2.00(1.24- \\
3.23)\end{array}$ & $\begin{array}{l}4.57 \\
(3.51- \\
5.93)\end{array}$ & $\begin{array}{l}1.73(1.10- \\
2.73)\end{array}$ & $\begin{array}{l}5.16 \\
(3.97- \\
6.69)\end{array}$ & $\begin{array}{l}1.63(1.05- \\
2.52)\end{array}$ \\
\hline $\begin{array}{l}\text { CPR self- } \\
\text { efficacy }\end{array}$ & $\begin{array}{l}5.07 \\
(3.82- \\
6.74)\end{array}$ & $\begin{array}{l}1.96(1.24- \\
3.09)\end{array}$ & $\begin{array}{l}4.39 \\
(3.36- \\
5.73)\end{array}$ & $\begin{array}{l}1.81(1.18- \\
2.77)\end{array}$ & $\begin{array}{l}4.59 \\
(3.56- \\
5.93)\end{array}$ & $\begin{array}{l}1.73(1.17- \\
2.55)\end{array}$ \\
\hline $\begin{array}{l}\text { CPR } \\
\text { knowledge }\end{array}$ & $\begin{array}{l}4.76 \\
(3.54- \\
6.42)\end{array}$ & $\begin{array}{l}2.32(1.59- \\
3.39)\end{array}$ & $\begin{array}{l}4.01 \\
(3.04- \\
5.29)\end{array}$ & $\begin{array}{l}2.12(1.50- \\
3.01)\end{array}$ & $\begin{array}{l}3.19 \\
(2.48- \\
4.12)\end{array}$ & $\begin{array}{l}1.46(1.06- \\
2.00)\end{array}$ \\
\hline \multicolumn{7}{|c|}{ Willingness to perform conventional CPR } \\
\hline \multicolumn{7}{|c|}{ Age (control factor: $20-40$ years old) } \\
\hline $\begin{array}{l}-<20 \\
\text { years old }\end{array}$ & NS & NS & $\begin{array}{l}0.21 \\
(0.06- \\
0.78)\end{array}$ & $\begin{array}{l}0.10(0.01- \\
0.77)\end{array}$ & NS & NS \\
\hline $\begin{array}{l}-41-60 \\
\text { years old }\end{array}$ & $\begin{array}{l}1.23 \\
(1.08- \\
1.41)\end{array}$ & NS & $\begin{array}{l}0.84 \\
(0.76- \\
0.93)\end{array}$ & NS & NS & NS \\
\hline $\begin{array}{l}\text { Awareness } \\
\text { of CPR }\end{array}$ & $\begin{array}{l}1.70 \\
(1.14- \\
2.55)\end{array}$ & NS & NS & NS & NS & NS \\
\hline $\begin{array}{l}\text { CPR } \\
\text { training }\end{array}$ & $\begin{array}{l}1.97 \\
(1.40- \\
2.78)\end{array}$ & NS & NS & NS & NS & NS \\
\hline $\begin{array}{l}\text { CPR } \\
\text { experience }\end{array}$ & $\begin{array}{l}1.40 \\
(1.08- \\
1.81)\end{array}$ & NS & NS & NS & NS & NS \\
\hline $\begin{array}{l}\text { Health } \\
\text { care } \\
\text { provider }\end{array}$ & $\begin{array}{l}1.89 \\
(1.43- \\
2.49)\end{array}$ & NS & NS & NS & NS & NS \\
\hline
\end{tabular}




\begin{tabular}{|c|c|c|c|c|c|c|}
\hline \multirow{3}{*}{$\begin{array}{l}\text { Associated } \\
\text { factors }\end{array}$} & \multicolumn{6}{|c|}{ Odds ratio $(95 \% \mathrm{Cl})$} \\
\hline & \multicolumn{2}{|c|}{ Family members } & \multicolumn{2}{|c|}{ Acquaintances } & \multicolumn{2}{|l|}{ Strangers } \\
\hline & Univariable & Multivariable & Univariable & Multivariable & Univariable & Multivariable \\
\hline $\begin{array}{l}\text { CPR self- } \\
\text { efficacy }\end{array}$ & $\begin{array}{l}1.87 \\
(1.44- \\
2.42)\end{array}$ & NS & $\begin{array}{l}1.40 \\
(1.05- \\
1.86)\end{array}$ & NS & NS & NS \\
\hline $\begin{array}{l}\text { CPR } \\
\text { knowledge }\end{array}$ & $\begin{array}{l}2.49 \\
(1.92- \\
3.23)\end{array}$ & $\begin{array}{l}2.16(1.59- \\
2.94)\end{array}$ & $\begin{array}{l}1.46 \\
(1.10- \\
1.94)\end{array}$ & NS & $\begin{array}{l}1.54 \\
(1.12- \\
2.11)\end{array}$ & NS \\
\hline
\end{tabular}

Participants were less willing to perform chest compression, AED, or mouth-to-mouth, mouth-to-face shield, or mouth-to-pocket mask ventilation during the pandemic in nearly all cases. However, lay participants' willingness to perform AED on family members and acquaintances did not differ (Table 4). While HCPs were less willing to perform chest compression in strangers, lay participants were not. Relationship with the victim was not correlated with willingness to perform AED in either group. In both groups, willingness to perform ventilation differed by relationship. However, the percentage of reductions during the pandemic in the HCP group was greater, except in the case of mouth-to-mouth ventilation on a family member. 
Table 4

Impact of the relationship between the bystander and victim on willingness to perform CPR maneuvers during the COVID-19 pandemic

\begin{tabular}{|c|c|c|c|c|c|c|}
\hline \multirow[t]{2}{*}{ Variable } & \multicolumn{3}{|c|}{ Laypersons $(\mathrm{N}=419)$} & \multicolumn{3}{|c|}{ Health care providers $(\mathrm{N}=716)$} \\
\hline & $\begin{array}{l}\text { Family } \\
\text { members }\end{array}$ & Acquaintances & Strangers & $\begin{array}{l}\text { Family } \\
\text { members }\end{array}$ & Acquaintances & Strangers \\
\hline \multicolumn{7}{|c|}{ Chest compression } \\
\hline $\begin{array}{l}\text { Non- } \\
\text { pandemic (n, } \\
(\%))\end{array}$ & $237(56.6)$ & $237(56.6)$ & $\begin{array}{l}216 \\
(51.6)\end{array}$ & $\begin{array}{l}669 \\
(93.4)^{b}\end{array}$ & $668(93.3)^{c}$ & $\begin{array}{l}615 \\
(85.9)\end{array}$ \\
\hline $\begin{array}{l}\text { Pandemic (n, } \\
(\%))\end{array}$ & $177(42.2)$ & $176(42.0)$ & $\begin{array}{l}150 \\
(35.8)\end{array}$ & $\begin{array}{l}587 \\
(82.0)^{b}\end{array}$ & $581(81.1)^{c}$ & $\begin{array}{l}506 \\
(70.7)\end{array}$ \\
\hline $\begin{array}{l}\text { Risk ratio } \\
(95 \% \mathrm{Cl})\end{array}$ & $\begin{array}{l}0.75 \\
(0.65- \\
0.86)\end{array}$ & $\begin{array}{l}0.74(0.65- \\
0.85)\end{array}$ & $\begin{array}{l}0.69 \\
(0.59- \\
0.81)\end{array}$ & $\begin{array}{l}0.88 \\
(0.84- \\
0.91)\end{array}$ & $\begin{array}{l}0.87(0.84- \\
0.91)\end{array}$ & $\begin{array}{l}0.82 \\
(0.78- \\
0.87)\end{array}$ \\
\hline \multicolumn{7}{|c|}{ Automated external defibrillator } \\
\hline $\begin{array}{l}\text { Non- } \\
\text { pandemic (n, } \\
(\%))\end{array}$ & $222(53.0)$ & $222(53.0)$ & $\begin{array}{l}210 \\
(50.1)\end{array}$ & $\begin{array}{l}645 \\
(90.1)\end{array}$ & $645(90.1)$ & $\begin{array}{l}628 \\
(87.7)\end{array}$ \\
\hline $\begin{array}{l}\text { Pandemic (n, } \\
(\%))\end{array}$ & $215(51.3)$ & $201(48.0)$ & $\begin{array}{l}175 \\
(41.8)\end{array}$ & $\begin{array}{l}591 \\
(82.5)\end{array}$ & $591(82.5)$ & $\begin{array}{l}563 \\
(78.6)\end{array}$ \\
\hline $\begin{array}{l}\text { Risk ratio } \\
(95 \% \mathrm{Cl})\end{array}$ & $\begin{array}{l}0.97 \\
(0.85-1.1)\end{array}$ & $\begin{array}{l}0.91(0.79- \\
1.04)\end{array}$ & $\begin{array}{l}0.83 \\
(0.72- \\
0.97)\end{array}$ & $\begin{array}{l}0.92 \\
(0.88- \\
0.96)\end{array}$ & $\begin{array}{l}0.92(0.88- \\
0.96)\end{array}$ & $\begin{array}{l}0.90 \\
(0.86- \\
0.94)\end{array}$ \\
\hline \multicolumn{7}{|c|}{ Mouth-to-mouth ventilation } \\
\hline $\begin{array}{l}\text { Non- } \\
\text { pandemic (n, } \\
(\%))\end{array}$ & $\begin{array}{l}161(38.4) \\
a\end{array}$ & $140(33.4)^{c}$ & $99(23.6)$ & $\begin{array}{l}380 \\
(53.1) \text { a, b }\end{array}$ & $268(37.4)^{c}$ & $\begin{array}{l}177 \\
(24.7)\end{array}$ \\
\hline $\begin{array}{l}\text { Pandemic (n, } \\
(\%))\end{array}$ & $50(11.9)^{a}$ & $34(8.1)$ & $25(6.0)$ & $\begin{array}{l}160 \\
(22.3)^{a, b}\end{array}$ & $60(8.4)^{c}$ & $33(4.6)$ \\
\hline $\begin{array}{l}\text { Risk ratio } \\
(95 \% \mathrm{Cl})\end{array}$ & $\begin{array}{l}0.31 \\
(0.23- \\
0.41)\end{array}$ & $\begin{array}{l}0.24(0.17- \\
0.34)\end{array}$ & $\begin{array}{l}0.25 \\
(0.17- \\
0.38)\end{array}$ & $\begin{array}{l}0.42 \\
(0.36- \\
0.49)\end{array}$ & $\begin{array}{l}0.22(0.17- \\
0.29)\end{array}$ & $\begin{array}{l}0.19 \\
(0.13- \\
0.27)\end{array}$ \\
\hline
\end{tabular}

\section{Face shield ventilation}

\footnotetext{
a statistically significant between family members and acquaintances $(p<0.05)$

${ }^{b}$ statistically significant between family members and strangers $(p<0.05)$

${ }^{c}$ statistically significant between acquaintances and strangers $(p<0.05)$
} 


\begin{tabular}{|c|c|c|c|c|c|c|}
\hline \multirow[t]{2}{*}{ Variable } & \multicolumn{3}{|c|}{ Laypersons $(\mathrm{N}=419)$} & \multicolumn{3}{|c|}{ Health care providers $(\mathrm{N}=716)$} \\
\hline & $\begin{array}{l}\text { Family } \\
\text { members }\end{array}$ & Acquaintances & Strangers & $\begin{array}{l}\text { Family } \\
\text { members }\end{array}$ & Acquaintances & Strangers \\
\hline $\begin{array}{l}\text { Non- } \\
\text { pandemic (n, } \\
(\%))\end{array}$ & $\begin{array}{l}150(35.8) \\
b\end{array}$ & $134(32.0)^{c}$ & $91(21.7)$ & $\begin{array}{l}404 \\
(56.4) a, b\end{array}$ & $327(45.7)^{c}$ & $\begin{array}{l}220 \\
(30.7)\end{array}$ \\
\hline $\begin{array}{l}\text { Pandemic (n, } \\
(\%))\end{array}$ & $\begin{array}{l}95(22.7) \\
a, b\end{array}$ & $72(17.2)^{c}$ & $48(11.5)$ & $\begin{array}{l}215 \\
(30.0)^{a, b}\end{array}$ & $106(14.8)^{c}$ & $67(9.4)$ \\
\hline $\begin{array}{l}\text { Risk ratio } \\
(95 \% \mathrm{Cl})\end{array}$ & $\begin{array}{l}0.63 \\
(0.51- \\
0.79)\end{array}$ & $\begin{array}{l}0.54(0.42- \\
0.69)\end{array}$ & $\begin{array}{l}0.53 \\
(0.38- \\
0.73)\end{array}$ & $\begin{array}{l}0.53 \\
(0.47- \\
0.61)\end{array}$ & $\begin{array}{l}0.32(0.27- \\
0.39)\end{array}$ & $\begin{array}{l}0.30 \\
(0.24- \\
0.39)\end{array}$ \\
\hline \multicolumn{7}{|c|}{ Pocket mask ventilation } \\
\hline $\begin{array}{l}\text { Non- } \\
\text { pandemic (n, } \\
(\%))\end{array}$ & $\begin{array}{l}220(52.5) \\
a, b\end{array}$ & $191(45.6)^{c}$ & $\begin{array}{l}136 \\
(32.5)\end{array}$ & $\begin{array}{l}540 \\
(75.4)^{a, b}\end{array}$ & $469(65.5)^{c}$ & $\begin{array}{l}347 \\
(47.8)\end{array}$ \\
\hline $\begin{array}{l}\text { Pandemic (n, } \\
(\%))\end{array}$ & $\begin{array}{l}168(40.1) \\
a, b\end{array}$ & $127(30.3)^{c}$ & $89(21.2)$ & $\begin{array}{l}316 \\
(44.1)^{a, b}\end{array}$ & $193(27.0)^{c}$ & $\begin{array}{l}129 \\
(18.0)\end{array}$ \\
\hline $\begin{array}{l}\text { Risk ratio } \\
(95 \% \mathrm{Cl})\end{array}$ & $\begin{array}{l}0.76 \\
(0.66- \\
0.89)\end{array}$ & $\begin{array}{l}0.66(0.56- \\
0.8)\end{array}$ & $\begin{array}{l}0.65 \\
(0.52- \\
0.82)\end{array}$ & $\begin{array}{l}0.59 \\
(0.53- \\
0.64)\end{array}$ & $\begin{array}{l}0.41(0.36- \\
0.47)\end{array}$ & $\begin{array}{l}0.38 \\
(0.32- \\
0.45)\end{array}$ \\
\hline \multicolumn{7}{|c|}{ a statistically significant between family members and acquaintances $(p<0.05)$} \\
\hline \multicolumn{7}{|c|}{ b statistically significant between family members and strangers $(p<0.05)$} \\
\hline
\end{tabular}

\section{Discussion}

Our study population represents urban residents who are able to participate in an online survey. Most were female, had a bachelor's degree, and worked full time. The mean age was about 40 years. We found that $65.4 \%$ of lay participants had heard of bystander CPR, compared to about $90 \%$ found in studies in other Asian countries. ${ }^{9-10,12}$ This suggests the need for better CPR recognition in Thailand. However, the $45.6 \%$ of lay participants who had taken a CPR course was comparable with the findings of previous studies. ${ }^{9-10,12}$ Two-thirds of HCPs and $13.6 \%$ of lay participants expressed CPR self-efficacy, which may affect their willingness to perform CPR.

These results show that the COVID-19 pandemic has reduced the willingness of bystanders to perform compression-only or conventional CPR in Thailand, despite it being a low-risk country. By contrast, a previous systematic review could not provide a firm conclusion as to the effect of COVID-19 on bystanders' willingness to perform CPR. While studies from Western countries have shown lower rates of bystander CPR, 
results from Asian countries have been inconsistent. ${ }^{17,21-23}$ The severity of the situation might affect willingness, but further study is required to determine whether this is the case.

We found that the pandemic had a greater impact on participants' willingness to perform conventional CPR (50\% reduction) than compression-only CPR. Similar reductions were seen in the willingness to perform mouth-to-mouth, mouth-to-face mask, or mouth-to-pocket mask ventilation. Although the percentage of reduction in HCPs was greater than in lay participants, willingness to perform ventilation was comparable between the two groups. The fact that ventilation is an aerosol generation procedure (AGP), might cause participants (especially HCPs) to be wary of the risk of COVID transmission. Similarly, the lack of clarity as to whether chest compressions and defibrillation are AGPs may have affected participants' willingness to perform these procedures. ${ }^{24}$ Although our study took place during a period in which Thailand had few COVID cases. New cases in each day were between 0-22 cases. However, the lack of an available vaccine may have contributed to participants' reluctance.

We also found that the participant's relationship to the victim affected their willingness to perform CPR. While lay participants were equally willing to perform conventional CPR on family members and acquaintances, this was not true of HCPs, possibly out of fear of transmission. This suggests that relationship to the victim may be a more significant factor for HCPs than laypeople. Participants in both groups were least likely to perform CPR on strangers, which is consistent with other studies.

Overall willingness to perform CPR (pandemic and non-pandemic) in our study was lower than in previous studies. ${ }^{7,9-11}$ This may have been partly due to insufficient CPR knowledge and low CPR self-efficacy in lay participants. A cultural fear of making mistakes may have been another factor, as may particularly laws of a country and citizens' awareness of them. In some Western countries, it is fairly well known that Good Samaritan laws protect bystanders who perform CPR, whereas a previous study in Hong Kong showed that only $12.1 \%$ of the population were aware of a Good Samaritan Law concept. ${ }^{25}$ While Good Samaritan law is enforced in Thailand in the year 2015.

Factors associated with willingness to perform compression-only CPR in our study were being an $\mathrm{HCP}$, having high CPR self-efficacy, and having good CPR knowledge. Being single was also associated with higher willingness, but only with family members and acquaintances. However, good CPR knowledge was the only factor associated with willingness to perform conventional CPR. Despite this, being an HCP (roughly half of whom had good CPR knowledge) was not associated. Younger participants and students were less willing to perform compression-only CPR. However, our sample size was not large enough to determine a conclusive association. We did not find any correlation with gender, marriage status, education, work status, or experience with CPR training, as have previous studies. ${ }^{8,9,13-14}$

Our results showed that $70-90 \%$ of participants would refuse to perform conventional CPR during the pandemic, and $60-95 \%$ would refuse to perform ventilation despite including a description of the ventilation technique and its benefits in the survey. In HCPs, $72.2-82.5 \%$ were willing to perform compression-only CPR compared to $17.3-35.1 \%$ for conventional CPR. Strategies to increase the rate of bystander CPR should focus on how people view interpersonal relationships, culture, and differences between HCPs and laypeople. 
Adopting CPR techniques that limit COVID-19 transmission might be a way to increase the willingness of Thai bystanders to perform CPR.

One limitation in this study was selection bias, as those interested in CPR or with CPR knowledge were more likely to participate in the survey, thus affecting the results. In addition, willingness to perform CPR is a surrogate outcome that may not accurately reflect behavior in real-life situations. However, measuring the prevalence of actual CPR performance during the pandemic would have been prohibitively complex, so we selected willingness to perform bystander CPR as a representation. Furthermore, although a high percentage of the Thai population have internet access, the fact that our survey was conducted online means that our participants were likely disproportionately urban and in the middle to high socioeconomic class, as it required a smartphone or computer with reliable internet. Despite these limitations, our study provided data regarding the impact of the pandemic and the relationship between bystanders and the victim on willingness to perform CPR.

\section{Conclusion}

In summary, participants were less willing to perform CPR during the COVID-19 pandemic, except in cases in which the bystander is a layperson, and the victim is a family member. The relationship between bystander and victim had a more significant effect on willingness to perform conventional CPR than compression-only CPR, especially in HCPs. CPR knowledge, self-efficacy, single marital status, and being an HCP were associated with a higher willingness to perform CPR during the COVID-19 pandemic.

\section{Abbreviations}

AED: automated defibrillator; Cl: confidence interval; COVID-19: Coronavirus Disease 2019; CPR: cardiopulmonary resuscitation; HCPs: healthcare providers; OHCA: Out-of-hospital cardiac arrest; OR: Odds ratio; SD: Standard Deviation; STROBE: STrengthening the Reporting of OBservational studies in Epidemiology.

\section{Declarations}

\section{Acknowledgment}

We would like to acknowledge Dr. Dylan Southard for editing the MS via the KKU Publication Clinic (Thailand).

\section{Author's contributions}

BS, MT, BPI, and BPO conceived and designed the study. BS, MT, BPI, and BPO developed the study protocol. $\mathrm{BS}, \mathrm{MT}$, and BPO designed and tested the study instrument. BS, MT, BPI, and BPO supervised data collection. BS, MT, and BPO analyzed the data. BS, MT, BPI, and BPO prepared and approved the manuscript. 


\section{Funding}

None.

\section{Availability of data and materials}

The datasets used and analyzed during the current study are available from the corresponding author on reasonable request.

\section{Ethics approval and consent to participate}

The Institute Review Board (Centre for Ethics in Human Research, Khon Kaen University, Thailand) approved the study (registration number: HE631328). All participants were consenting to participate in this study before doing the questionnaire.

\section{Consent for publication}

Not applicable.

\section{Competing interests}

The authors declare that they have no competing interests.

\section{References}

1. Ong ME, Shin SD, De Souza NN, et al; PAROS Clinical Research Network. Outcomes for out-of-hospital cardiac arrests across 7 countries in Asia: The Pan Asian Resuscitation Outcomes Study (PAROS). Resuscitation. 2015;96:100-8. http://doi.org/10.1016/j.resuscitation.2015.07.026.

2. Lim SL, Smith K, Dyson K, et al. Incidence and Outcomes of Out-of-Hospital Cardiac Arrest in Singapore and Victoria: A Collaborative Study. J Am Heart Assoc. 2020;9:e015981. http://doi.org/10.1161/JAHA.119.015981.

3. Olasveengen TM, Mancini ME, Perkins GD, et al; Adult Basic Life Support Collaborators. Adult Basic Life Support: International Consensus on Cardiopulmonary Resuscitation and Emergency Cardiovascular Care Science With Treatment Recommendations. Resuscitation. 2020;156:A35-79. http://doi.org/10.1016/j.resuscitation.2020.09.010.

4. Olasveengen TM, Semeraro F, Ristagno G, et al. European Resuscitation Council Guidelines 2021: Basic Life Support. Resuscitation. 2021;161:98-114. http://doi.org/10.1016/j.resuscitation.2021.02.009.

5. Yan S, Gan Y, Jiang N, et al. The global survival rate among adult out-of-hospital cardiac arrest patients who received cardiopulmonary resuscitation: a systematic review and meta-analysis. Crit Care. 2020;24:61. http://doi.org/10.1186/s13054-020-2773-2.

6. Kiguchi T, Okubo M, Nishiyama C, et al. Out-of-hospital cardiac arrest across the World: First report from the International Liaison Committee on Resuscitation (ILCOR). Resuscitation. 2020;152:39-49. http://doi.org/10.1016/j.resuscitation.2020.02.044. 
7. Huang Q, Hu C, Mao J. Are Chinese Students Willing to Learn and Perform Bystander Cardiopulmonary Resuscitation? J Emerg Med. 2016;51:712-20. http://doi.org/10.1016/j.jemermed.2016.02.033.

8. Kua PHJ, White AE, Ng WY, et al. Knowledge and attitudes of Singapore schoolchildren learning cardiopulmonary resuscitation and automated external defibrillator skills. Singapore Med J. 2018;59:487-99. http://doi.org/10.11622/smedj.2018021.

9. Pei-Chuan Huang E, Chiang WC, et al. Public knowledge, attitudes and willingness regarding bystander cardiopulmonary resuscitation: A nationwide survey in Taiwan. J Formos Med Assoc. 2019;118:57281. http://doi.org/10.1016/j.jfma.2018.07.018.

10. Karuthan SR, Firdaus PJFB, Angampun ADG, et al. Knowledge of and willingness to perform HandsOnly cardiopulmonary resuscitation among college students in Malaysia. Medicine (Baltimore). 2019;98:e18466. http://doi.org/10.1097/MD.0000000000018466.

11. Son JW, Ryoo HW, Moon S, et al. Association between public cardiopulmonary resuscitation education and the willingness to perform bystander cardiopulmonary resuscitation: a metropolitan citywide survey. Clin Exp Emerg Med. 2017;4:80-7. http://doi.org/10.15441/ceem.16.160.

12. Chen M, Wang Y, Li X, et al. Public Knowledge and Attitudes towards Bystander Cardiopulmonary Resuscitation in China. Biomed Res Int. 2017;2017:3250485. http://doi.org/10.1155/2017/3250485.

13. Chiang WC, Ko PC, Chang AM, et al. Bystander-initiated CPR in an Asian metropolitan: does the socioeconomic status matter? Resuscitation. 2014;85:53-

8. http://doi.org/10.1016/j.resuscitation.2013.07.033.

14. Urban J, Thode H, Stapleton E, Singer AJ. Current knowledge of and willingness to perform Hands-Only CPR in laypersons. Resuscitation. 2013;84:1574-8. http://doi.org/10.1016/j.resuscitation.2013.04.014.

15. Hawkes CA, Brown TP, Booth S, et al. Attitudes to Cardiopulmonary Resuscitation and Defibrillator Use: A Survey of UK Adults in 2017. J Am Heart Assoc.

2019;8:e008267.http://doi.org/10.1161/JAHA.117.008267.

16. Cho JW, Jung $\mathrm{H}$, Lee MJ, et al. Preparedness of personal protective equipment and implementation of new CPR strategies for patients with out-of-hospital cardiac arrest in the COVID-19 era. Resusc Plus. 2020;3:100015.http://doi.org/10.1016/j.resplu.2020.100015.

17. Scquizzato T, Landoni G, Paoli A, et al. Effects of COVID-19 pandemic on out-of-hospital cardiac arrests: A systematic review. Resuscitation. 2020;157:241-7.

http://doi.org/10.1016/j.resuscitation.2020.10.020.

18. Grunau B, Bal J, Scheuermeyer F, et al. Bystanders are less willing to resuscitate out-of-hospital cardiac arrest victims during the COVID-19 pandemic. Resusc Plus. 2020;4:100034.

http://doi.org/10.1016/j.resplu.2020.100034.

19. Kim HS, Sherman DK, Taylor SE. Culture and social support. Am Psychol. 2008;63:51826.http://doi.org/10.1037/0003-066X.

20. Orehek E, Forest AL, Barbaro N. A People-as-Means Approach to Interpersonal Relationships. Perspect Psychol Sci. 2018;13:373-89. http://doi.org/10.1177/1745691617744522.

21. Howell S, Nehme Z, Eastwood K, Battaglia T, Buttery A, Bray J. The impact of COVID-19 on the Australian public's willingness to perform hands-only CPR. Resuscitation. 2021;163:26-7. 
http://doi.org/10.1016/j.resuscitation.2021.03.034.

22. Mathew S, Harrison N, Chalek AD, et al. Effects of the COVID-19 pandemic on out-of-hospital cardiac arrest care in Detroit. Am J Emerg Med. 2021;46:90-6. http://doi.org/10.1016/j.ajem.2021.03.025.

23. Nishiyama C, Kiyohara K, Iwami T, et al. Influence of COVID-19 pandemic on bystander interventions, emergency medical service activities, and patient outcomes in out-of-hospital cardiac arrest in Osaka City, Japan. Resusc Plus. 2021;5:100088. http://doi.org/10.1016/j.resplu.2021.100088.

24. Couper K, Taylor-Phillips S, Grove A, et al. COVID-19 in cardiac arrest and infection risk to rescuers: A systematic review. Resuscitation. 2020;151:59-66. http://doi.org/10.1016/j.resuscitation.2020.04.022.

25. Hung KK, Leung C, Siu A, Graham CA. Good Samaritan Law and bystander cardiopulmonary resuscitation: Cross-sectional study of 1223 first-aid learners in Hong Kong. Hong Kong J Emerg Med. 2021;28:22-9. http://doi.org/10.1177/1024907919870928

\section{Supplementary Files}

This is a list of supplementary files associated with this preprint. Click to download.

- 3.supplementmaterialPaperbasedcopyoftheonlinequestionnaireEnglishversion.pdf 